\title{
The influence of maternal size on pre- and postnatal growth in the horse: III Postnatal growth
}

\author{
W R Allen, Sandra Wilsher, Clare Tiplady and R M Butterfield ${ }^{1}$ \\ University of Cambridge, Department of Clinical Veterinary Medicine Equine Fertility Unit, Mertoun Paddocks, \\ Woodditton Road, Newmarket, Suffolk CB8 9BH, UK and 56 Queens Road, Lawson, New South Wales 2783, \\ Australia
}

Correspondence should be addressed to $W R$ Allen

\begin{abstract}
The growth parameters exhibited by seven Thoroughbred (Tb) foals that had experienced a 'restricted' in utero existence following transfer as embryos to the uteri of smaller Pony (P) mares (Tb-in-P) and, conversely, six $\mathbf{P}$ foals that experienced a 'luxurious' in utero existence after transfer to larger Tb mares (P-in-Tb), were compared from birth to 3 years of age with those exhibited by six normal Tb-in-Tb and six P-in-P foals conceived by within-breed artificial insemination. Bodyweight, height at the withers, girth, poll-to-nose length, crown-rump length and three foreleg longbone measurements were made at regular intervals. At birth, an approximate $15 \%$ reduction or increase in parameters was observed in the $\mathrm{Tb}$-in-P and $\mathrm{P}$-in- $\mathrm{Tb}$ respectively, which declined to $5 \%$ by 3 years of age. Growth post partum was affected by restricted or enhanced growth in utero. In the first 6 months post partum, growth rate was enhanced in the previously restricted Tb-in-P foals and curbed in the previously enhanced P-in-Tb foals compared with their respective controls. Overall, the similarity of the responses of the offspring to both 'restriction' and 'luxury' in utero ensured that no major changes to conformation resulted from either treatment. Thus, the Thoroughbreds carried by the Pony mares were merely scaled down versions of the Tb-in-Tb controls while the Ponies carried by the Thoroughbred mares were scaled up versions of the P-in-P controls.
\end{abstract}

Reproduction (2004) 127 67-77

\section{Introduction}

Walton \& Hammond (1938) used artificial insemination to reciprocally cross Shire horses and Shetland ponies, and thereby demonstrated a profound influence of maternal size on pre- and postnatal growth of the foal. More recently, Tischner and his colleagues similarly highlighted the influence of maternal size upon foal growth when they used embryo transfer to gestate Polish Konig pony foals in the uteri of larger draught-type mares (Tischner 1985, 1987, Tischner \& Klimczak 1989).

To examine the hypothesis that changes in uterine size may significantly and permanently alter the rate and form of fetal and postnatal growth of the foal, we too have employed embryo transfer between larger Thoroughbred (Tb) and smaller Pony (P) mares to create two groups of between-breed gestated foals for direct comparison with their within-breed counterparts conceived by artificial insemination. We reported previously on striking maternal blood endocrinological differences between these four types of pregnancy (Allen et al. 2002b) and on the highly significant correlations between foal birthweight and the gross area, weight and volume of the allantochorion.
We also highlighted the strong correlation between maternal genotype and the surface density of microcotyledons on the external surface of the allantochorion and the contribution of this parameter to the total microscopic area of fetomaternal contact at the placental interface (Allen et al. 2002a).

In this paper we present the growth curves for bodyweight, height, crown-rump (CR) length and five other linear measurements made on the four groups of offspring between birth and 3 years of age. We use these data to determine whether uterine environment can restrict or exaggerate postnatal growth of the foal, and whether foals that suffer growth retardation in utero exhibit significant catch-up growth postnatally.

\section{Materials and Methods}

\section{Experimental animals}

Following embryo recovery performed non-surgically on day 7 after ovulation in the donor mares and non-surgical transfer of the embryos to synchronised recipient mares of the other breed (Allen 1982), eight Tb-in-P foals were 
born after a mean \pm S.E.M. gestation of $332 \pm 2.8$ days and seven P-in-Tb foals were born after $331 \pm 2.7$ days of gestation. Following conventional within-breed artificial insemination, seven $\mathrm{Tb}$-in-Tb foals were born after a mean \pm S.E.M. gestation of $339 \pm 3.0$ days and seven $\mathrm{P}$-in-P foals were born after a gestation of $325 \pm 3.0$ days. All the foals were born during Spring and early Summer (April-June inclusive) in 2 successive years (1997 and 1998) and they were weaned by removing their mothers from the group when they reached 6 months of age.

The mean \pm S.E.M. weight prior to the start of pregnancy of the $14 \mathrm{~Tb}$ mares used as embryo donors and surrogate mothers was $589.1 \pm 18.4 \mathrm{~kg}$ while that of the $15 \mathrm{P}$ mares was $327.8 \pm 19.7 \mathrm{~kg}$. One Tb stallion weighing $514 \mathrm{~kg}$ and one $\mathrm{P}$ stallion weighing $396 \mathrm{~kg}$ were used as semen donors.

\section{Measurement schedules}

The foals were weighed within $1 \mathrm{~h}$ after birth before they had nursed from their mothers. The placenta was collected at spontaneous third stage labour from each mare and subjected to a range of gross and microscopic measurements, as described by Allen et al. (2002a).

Each foal was weighed on the day after birth, then at fortnightly intervals to weaning at 6 months of age, and thereafter at monthly intervals to 3 years of age, using a large animal weighbridge (Salter Universal Weighing Machine; Salter Ltd, Malton, North Yorks, UK). Height at the withers was measured with an adjustable measuring stick while the animal was standing evenly on a level concrete floor. Six body and longbone measurements were then made using a flexible tape measure as illustrated in Fig. 1. These measurements were: (i) poll-to-nose; the distance from the point of the nuchal crest of the occipital bone between the ears down the dorsal surface of the face to the upper lip; (ii) CR length; the distance from the highest point of the nuchal crest of the occipital bone to the base of the tail; (iii) girth; the circumference of the chest immediately caudal to the withers; (iv) elbow-to-knee; the distance from the point of the elbow (olecranon process) to the accessory carpal bone on the lateral surface of the nearside foreleg; (v) knee-to-fetlock; the distance from the point of contact of the intermediate and fourth carpal bones to the proximal condyle of the first phalanx on the lateral surface of the nearside foreleg; (vi) cannon circumference; the circumference of the cannon bone and associated soft tissues measured immediately distal to the metacarpal tuberosity at the proximal end of the cannon bone.

\section{Horse management}

The 29 foals were raised in two groups, one of 14 fillies and the other of 15 colts. During the colder winter months (November-March inclusive) they were housed at night in large yards deep-littered with straw where they were fed a ration of cubed concentrates night and morning and were given good quality hay and water ad libitum. They were turned out to graze for approximately $6 \mathrm{~h} /$ day.

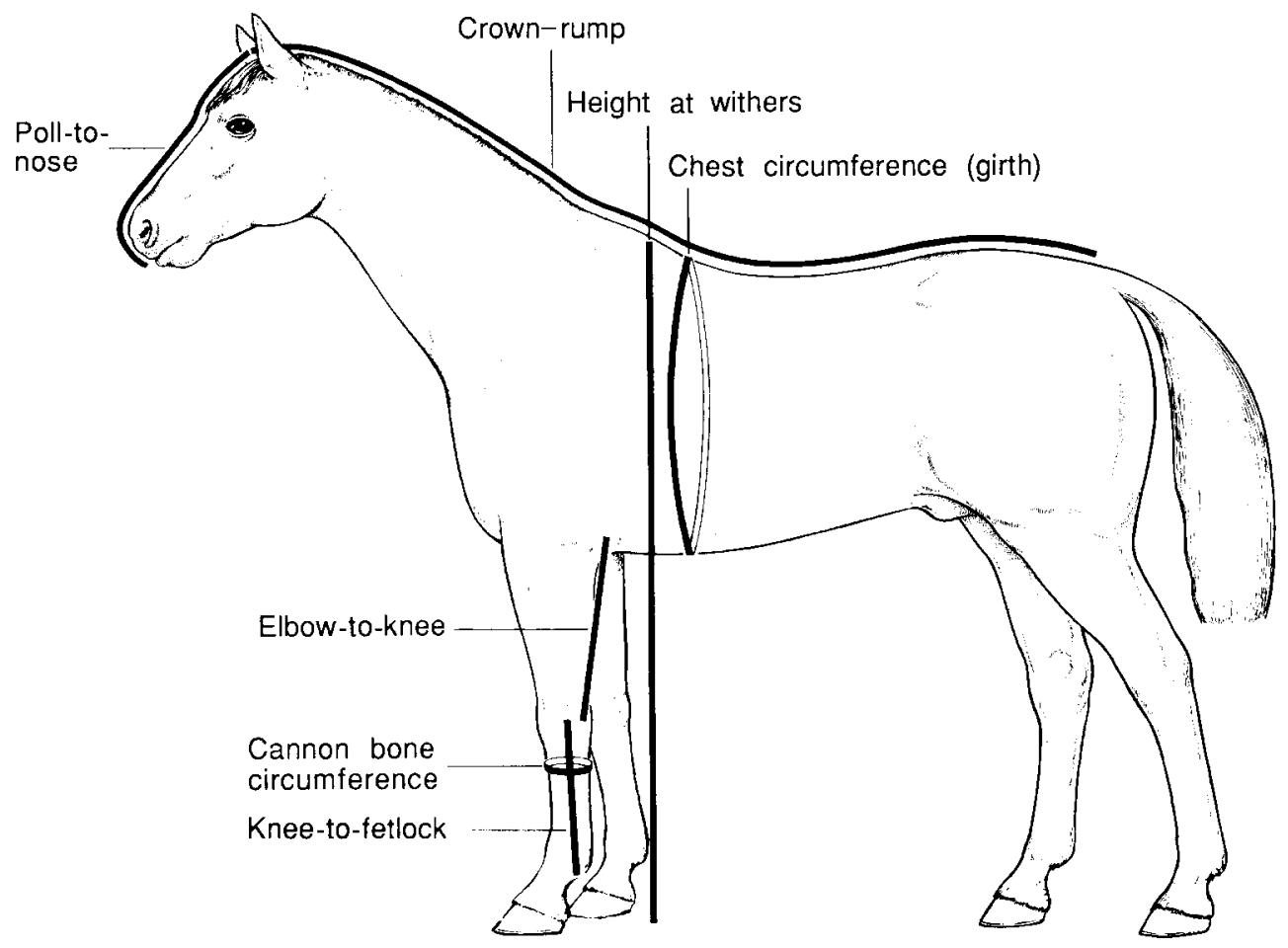

Figure 1 Diagrammatic representation of the seven head, body and longbone measurements made from birth to 36 months of age. 
During the warm summer months (April-October) grazing was available $24 \mathrm{~h} /$ day and they were occasionally supplemented with hay and a small allocation of the cubed concentrates depending on the quality of the pasture. They were wormed regularly by oral administration of proprietary anthelmintics and the paddocks were 'swept' regularly to remove the accumulating dung. The animals were vaccinated annually against influenza and tetanus and the colts were all castrated in March when they were between 19 and 21 months old.

Four animals, one from each group, died before 3 years of age. One Tb-in-P filly was destroyed on humane grounds at 21 months of age due to severe inward rotation and misalignment of the cannon bone in the offside foreleg which caused increasing lameness as her bodyweight increased. One $\mathrm{P}$-in-Tb colt, one $\mathrm{P}$-in-P filly and one $\mathrm{Tb}$-in-Tb colt were shot when they contracted acute equine dysautonomia ('grass sickness'; Whitwell 1997) at, respectively, 28, 22 and 15 months of age. The measurements for these four animals were removed from the calculations to leave six P-in-Tb ('luxurious') animals that were compared with six $\mathrm{P}$-in-P controls and seven Tb-in- $\mathrm{P}$ ('restricted') foals that were compared with six Tb-in-Tb controls, all from birth to 3 years of age.

\section{Statistical analysis}

Means \pm S.E.M. are presented throughout. Statistical analyses were performed using one- and two-way ANOVA with Student-Newman-Keuls method post hoc test (Sigma Stat v.2.0 program; SPSS Inc, Chicago, IL, USA). Differences were considered significant at $P<0.05$. The 'treatment' arrangement corresponded to a $2 \times 2$ factorial structure with $\mathrm{Tb}$ and $\mathrm{P}$ animals acting as donors for one factor and as recipients for the second factor. Eight anatomical features were measured regularly between birth and 36 months and the data were analysed using the method of summary statistics as suggested by Rowell \& Walters (1976) and Walters \& Rowell (1982). Conventional two-way ANOVA was carried out at each time-point and the changes over time were studied by analysing appropriate linear functions of the variables, the outline ANOVA being exactly as for the individual variables. The factorial analysis is able to examine the main effect of donor (Tb vs $\mathrm{P}$ ) and recipient (Tb vs $\mathrm{P}$ ), but also the interaction of these two effects. A significant interaction would indicate that the effect of the donor varied depending on the type of recipient. The absence of interaction leads to a simpler interpretation based on the main effects of donor and recipient.

\section{Results}

There was no evidence of an interaction effect of donor and recipient in any of the variables investigated. Accordingly, interpretation was based entirely on the main effects.

\section{Tb-in-P 'restricted' foals}

The mean growth curves exhibited by the Tb-in-P ('restricted') foals and their Tb-in-Tb controls for bodyweight, height at the withers, CR length, poll-to-nose length, girth, and for the three foreleg longbone measurements (elbow-to-knee, knee-to-fetlock and cannon bone circumference) are shown in Fig. 2. The Ponderal index $\left(k=\right.$ bodyweight/CR length ${ }^{3}$; Thompson 1942) is shown in Fig. 3. Absolute measurements at birth and 36 months, and the percentage increases over this time-period are shown in Table 1a. Photographs comparing 'restricted' Tb-in-P offspring with their Tb-in-Tb controls at 14 and 36 months of age are shown in Fig. 5a and b.

The mean growth curve profiles for all eight measurements were essentially comparable with each other between the two groups of offspring, with that for the 'restricted' foals lying below the control Tb-in-Tb curve (Fig. 2). In each case, the profiles increased steeply from birth to weaning around 20 weeks of age after which the rate of increase tended to slow to around 80-100 weeks and thereafter level off to the end of the recording period at 160 weeks. With both longbone measurements (elbowto-knee and knee-to-fetlock), however, a definite spurt in growth occurred between 60 and 90 weeks of age (Fig. $2 \mathrm{~g}$ and h). The mean \pm S.E.M. ages at which these two longbones, the radius (elbow-to-knee) and cannon (knee-tofetlock), stopped increasing in length, thereby indicating closure of the growth plates, were $80.0 \pm 1.7$ and $81.1 \pm 11.4$ weeks respectively for the 'restricted' offspring versus $72.7 \pm 4.3$ and $75.3 \pm 7.5$ weeks for their controls.

The initial sharp rise in the Ponderal index during the first month of life was less pronounced in the 'restricted' foals than in the Tb-in-Tb controls. The curves remained relatively parallel to each other until 60 weeks of age where they crossed one another and thereafter continued in an essentially parallel manner to 140 weeks. However, as they approached maturity the 'restricted' group acquired more weight for their length, as reflected in a greater Ponderal index (Fig. 3, upper graph).

\section{P-in-Tb ('luxurious') foals}

The mean growth curves displayed by the P-in-Tb ('luxurious') foals and their P-in-P controls are shown in Fig. 4. The Ponderal index curves are shown in Fig. 3 (lower graph). Absolute measurements at birth and 36 months of age and the percentage increases over the period are shown in Table 1a. Photographs comparing 'luxurious' and control offspring at 14 months and 3 years of age are given in Fig. 5c and d.

The mean profiles for bodyweight, CR length, height at the withers, head length and girth were essentially comparable with one another between the two groups, showing the initial steep rise to weaning at 20 weeks followed by a slower rate of increase thereafter to a levelling off around 100-120 weeks (Fig. 4a-e). Whereas the curves 


$$
a=\text { Tb-in- } T b \quad==\text { Tb-in-P }
$$
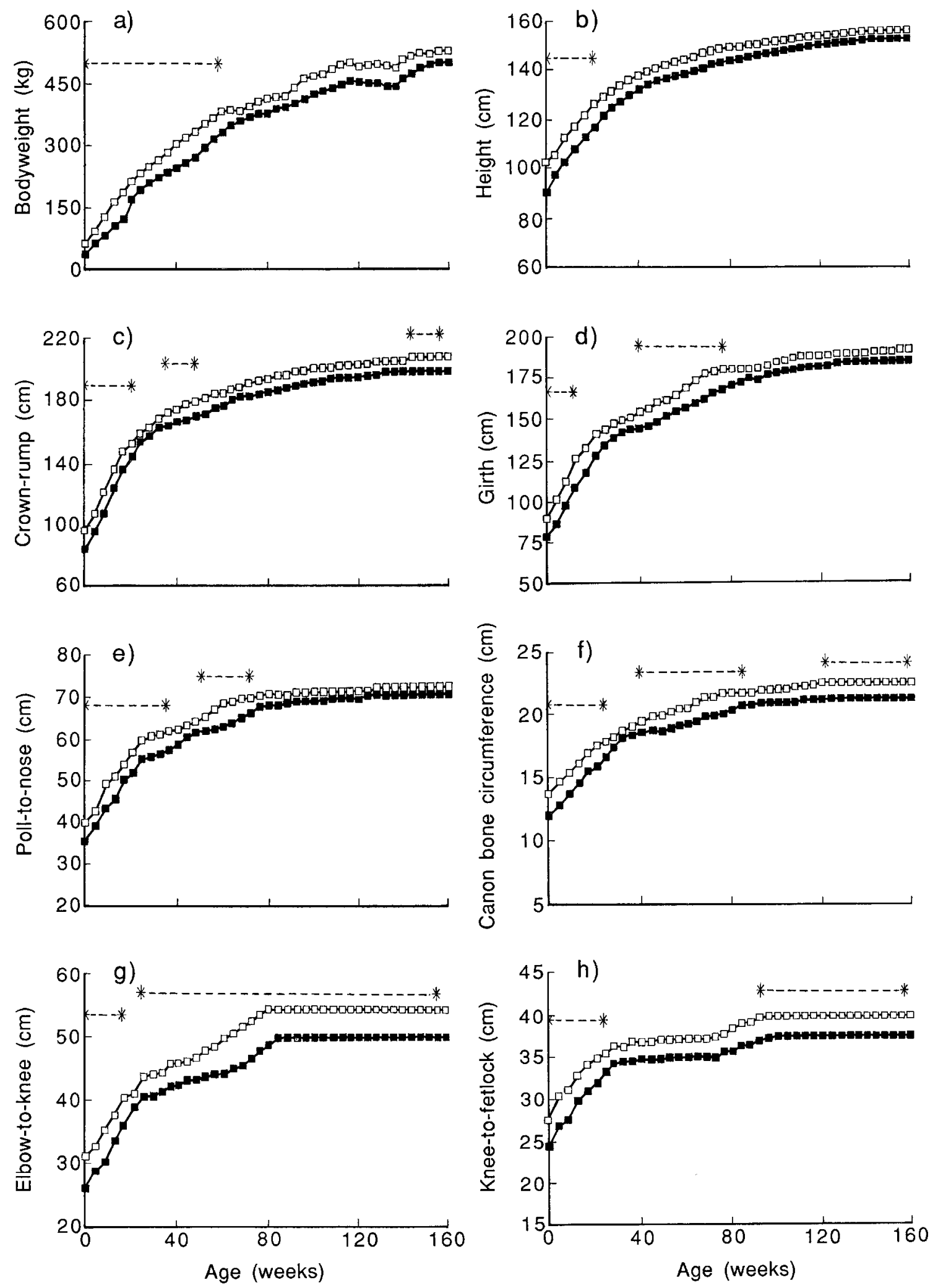

Figure 2 The mean growth curves exhibited by the Tb-in-P ('restricted'; $\mathbf{\square})$ animals and their Tb-in-Tb controls ( $\square$ ) for (a) bodyweight, (b) height at the withers, (c) CR length, (d) girth (chest circumference), (e) poll-to-nose length, (f) cannon bone circumference, (g) elbow-to-knee length and $(\mathrm{h})$ knee-to-fetlock length. The mean values between the asterisks differ significantly $(P<0.05)$ from each other. 

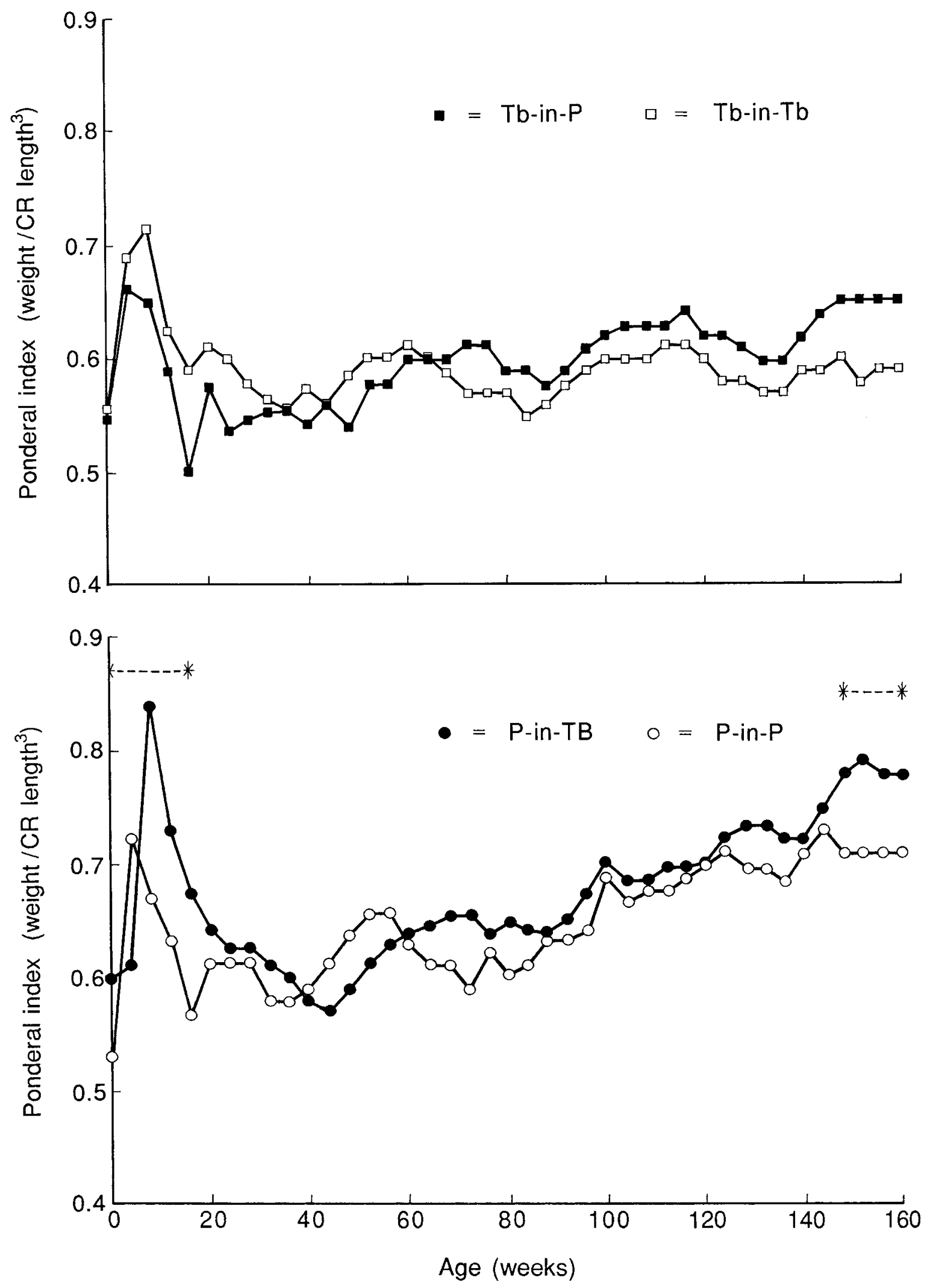

Figure 3 The Ponderal index $\left(k=\right.$ bodyweight/CR length ${ }^{3}$ ) for (upper) the Tb-in-P ('restricted') animals relative to their Tb-in-Tb controls and (lower) the P-in-Tb ('luxurious') animals relative to their P-in-P controls. The mean values between the asterisks differ significantly $(P<0.05)$ from each other. 
Table 1 (a) Mean \pm S.E.M. measurements of eight selected physical parameters at birth and 36 months of age, and the percentage increase between these two figures, for the four groups of foals. (b) Mean \pm S.E.M. for a two-way ANOVA with Tb and P acting as donors for one factor and as recipients for the second factor.

\begin{tabular}{|c|c|c|c|c|c|c|c|c|}
\hline \multirow[b]{3}{*}{ Parameter } & \multicolumn{4}{|l|}{ (a) } & \multicolumn{4}{|l|}{ (b) } \\
\hline & \multicolumn{2}{|c|}{ Restricted vs control } & \multicolumn{2}{|c|}{ Luxurious vs control } & \multicolumn{2}{|c|}{ Donor } & \multicolumn{2}{|c|}{ Recipient } \\
\hline & Tb-in-P & Tb-in-Tb & P-in-Tb & P-in-P & $\mathrm{Tb}$ & Pony & $\mathrm{Tb}$ & Pony \\
\hline \multicolumn{9}{|l|}{ Weight (kg) } \\
\hline Birth & $32.4 \pm 2.7$ & $51.4 \pm 2.4 * * *$ & $38.2 \pm 1.7$ & $24.2 \pm 1.5^{* * *}$ & $41.9 \pm 2.0$ & $29.5 \pm 2.0^{* * *}$ & $44.8 \pm 2.0$ & $26.6 \pm 2.0^{* * *}$ \\
\hline 36 months & $483.4 \pm 17.0$ & $514.8 \pm 13.3$ & $448.2 \pm 25.7$ & $388.8 \pm 19.6 *$ & $499.1 \pm 13.4$ & $418 \pm 13.9 * * *$ & $481.5 \pm 13.9$ & $436.1 \pm 13.4 *$ \\
\hline \multirow{2}{*}{\multicolumn{9}{|c|}{ Height $(\mathrm{cm})$}} \\
\hline & & & & & & & & \\
\hline Birth & $90.1 \pm 2.2$ & $102.1 \pm 1.0 * * *$ & $88.5 \pm 2.3$ & $77.5 \pm 2.3 * * *$ & $95.8 \pm 1.4$ & $82.8 \pm 1.5^{* * *}$ & $95.6 \pm 1.5$ & $84.0 \pm 1.4 * * *$ \\
\hline 36 months & $150.2 \pm 1.7$ & $153.3 \pm 1.1$ & $136.3 \pm 2.7$ & $132.2 \pm 1.8$ & $151.7 \pm 1.3$ & $134.3 \pm 1.4 * * *$ & $144.8 \pm 1.4$ & $141.2 \pm 1.3$ \\
\hline$\%$ increase & $67.5 \pm 5.1$ & $50.3 \pm 1.8^{*}$ & $54.2 \pm 1.8$ & $71.3 \pm 5.5^{*}$ & $58.9 \pm 2.8$ & $62.8 \pm 3.0$ & $52.3 \pm 3.0$ & $69.4 \pm 2.8^{* * *}$ \\
\hline \multicolumn{9}{|l|}{ CR length $(\mathrm{cm})$} \\
\hline Birth & $84.4 \pm 1.1$ & $97.3 \pm 1.3^{* * *}$ & $85.5 \pm 3.0$ & $77.0 \pm 2.5 *$ & $90.9 \pm 1.4$ & $81.3 \pm 1.5^{* * *}$ & $91.4 \pm 1.5$ & $80.7 \pm 1.4 * * *$ \\
\hline 36 months & $195.3 \pm 2.5$ & $205.7 \pm 2.2 *$ & $179.3 \pm 4.0$ & $175.5 \pm 3.8$ & $200.5 \pm 2.2$ & $177.4 \pm 2.3^{* * *}$ & $192.5 \pm 2.3$ & $185.4 \pm 2.2 *$ \\
\hline$\%$ increase & $131.4 \pm 2.7$ & $112.6 \pm 3.5 *$ & $110.7 \pm 7.2$ & $128.5 \pm 5.4 *$ & $122.0 \pm 3.4$ & $119.6 \pm 3.5$ & $111.7 \pm 3.5$ & $130.0 \pm 3.4 * * *$ \\
\hline \multicolumn{9}{|l|}{ Girth (cm) } \\
\hline Birth & $76.9 \pm 2.1$ & $87.5 \pm 1.2 * *$ & $80.5 \pm 3.4$ & $71.0 \pm 2.0^{*}$ & $82.2 \pm 1.6$ & $75.8 \pm 1.6^{* *}$ & $84.0 \pm 1.6$ & $73.9 \pm 1.6^{* * *}$ \\
\hline 36 months & $182.4 \pm 1.4$ & $188.0 \pm 1.9$ & $181.8 \pm 2.8$ & $176.8 \pm 3.2$ & $185.2 \pm 1.7$ & $179.3 \pm 1.7 *$ & $184.9 \pm 1.7$ & $179.6 \pm 1.7 *$ \\
\hline$\%$ increase & $138.3 \pm 5.8$ & $115.1 \pm 1.0$ & $127.3 \pm 6.8$ & $150.0 \pm 8.1$ & $126.7 \pm 4.4$ & $138.6 \pm 4.6$ & $121.2 \pm 4.6$ & $144.1 \pm 4.4^{* *}$ \\
\hline \multicolumn{9}{|l|}{ Poll-nose $(\mathrm{cm})$} \\
\hline Birth & $35.1 \pm 1.1$ & $39.2 \pm 0.6^{* *}$ & $33.3 \pm 1.1$ & $31.3 \pm 1.3$ & $37.2 \pm 0.7$ & $32.3 \pm 0.8^{* * *}$ & $36.3 \pm 0.8$ & $33.2 \pm 0.7 * *$ \\
\hline 36 months & $71.0 \pm 0.5$ & $72.0 \pm 0.5$ & $65.0 \pm 1.2$ & $63.5 \pm 1.5$ & $71.5 \pm 0.7$ & $64.3 \pm 0.7^{* * *}$ & $68.5 \pm 0.7$ & $67.3 \pm 0.7$ \\
\hline$\%$ increase & $49.5 \pm 1.6$ & $54.5 \pm 1.2$ & $51.3 \pm 1.2$ & $49.4 \pm 2.0$ & $52.0 \pm 1.1$ & $50.3 \pm 1.1$ & $52.9 \pm 1.1$ & $49.5 \pm 1.1 *$ \\
\hline \multicolumn{9}{|c|}{ Cannon circumference $(\mathrm{cm})$} \\
\hline Birth & $11.9 \pm 0.5$ & $13.3 \pm 0.4 *$ & $12.7 \pm 0.2$ & $11.2 \pm 0.4 *$ & $12.6 \pm 0.3$ & $11.9 \pm 0.3$ & $13.0 \pm 0.3$ & $11.5 \pm 0.3^{* * *}$ \\
\hline 36 months & $20.7 \pm 0.3$ & $21.8 \pm 0.3 *$ & $19.7 \pm 0.3$ & $18.7 \pm 0.3 *$ & $18.3 \pm 0.3$ & $16.7 \pm 0.3^{* * *}$ & $17.8 \pm 0.3$ & $17.2 \pm 0.3^{* *}$ \\
\hline$\%$ increase & $76.1 \pm 6.6$ & $64.5 \pm 5.5$ & $55.3 \pm 2.2$ & $68.1 \pm 5.7$ & $70.3 \pm 3.8$ & $61.7 \pm 3.9$ & $59.9 \pm 3.9$ & $72.1 \pm 3.8^{*}$ \\
\hline \multicolumn{9}{|c|}{ Elbow-knee $(\mathrm{cm})$} \\
\hline Birth & $25.7 \pm 0.4$ & $30.8 \pm 0.3^{* * *}$ & $27.5 \pm 0.7$ & $22.5 \pm 0.5^{* * *}$ & $28.3 \pm 0.3$ & $25.0 \pm 0.4 * * *$ & $29.2 \pm 0.4$ & $24.1 \pm 0.3^{* * *}$ \\
\hline 36 months & $49.9 \pm 0.4$ & $53.5 \pm 0.5^{* * *}$ & $47.2 \pm 0.6$ & $43.0 \pm 0.9^{* * *}$ & $51.7 \pm 0.4$ & $45.1 \pm 0.4^{* * *}$ & $50.3 \pm 0.4$ & $46.4 \pm 0.4^{* * *}$ \\
\hline$\%$ increase & $94.0 \pm 1.8$ & $73.6 \pm 2.4 * * *$ & $72.1 \pm 4.8$ & $91.3 \pm 3.2 * * *$ & $83.8 \pm 2.2$ & $81.7 \pm 2.3$ & $72.8 \pm 2.3$ & $92.6 \pm 2.2 * * *$ \\
\hline \multicolumn{9}{|c|}{ Knee-fetlock (cm) } \\
\hline Birth & $24.4 \pm 0.4$ & $27.5 \pm 0.8^{* * *}$ & $22.2 \pm 0.5$ & $20.0 \pm 0.6 *$ & $26.0 \pm 0.4$ & $21.1 \pm 0.4 * * *$ & $24.8 \pm 0.4$ & $22.2 \pm 0.4 * * *$ \\
\hline 36 months & $37.1 \pm 0.5$ & $40.8 \pm 0.4 * * *$ & $34.3 \pm 0.6$ & $32.2 \pm 0.7 *$ & $39.0 \pm 0.4$ & $33.3 \pm 0.4 * * *$ & $37.6 \pm 0.4$ & $34.7 \pm 0.4 * * *$ \\
\hline$\%$ increase & $52.3 \pm 2.8$ & $49.1 \pm 4.5$ & $55.1 \pm 3.1$ & $61.3 \pm 4.7$ & $50.7 \pm 2.6$ & $58.2 \pm 2.7$ & $52.1 \pm 2.7$ & $56.8 \pm 2.6$ \\
\hline
\end{tabular}

$* P<0.05,{ }^{* *} P<0.01,{ }^{* * *} P<0.001$ : in (a) significant differences between control and experimental pregnancies; in (b) between Tb and $\mathrm{P}$ genotypes as donor or recipients. 


$$
\text { - }=P \text {-in-TB } \quad 0=P \text {-in-P }
$$
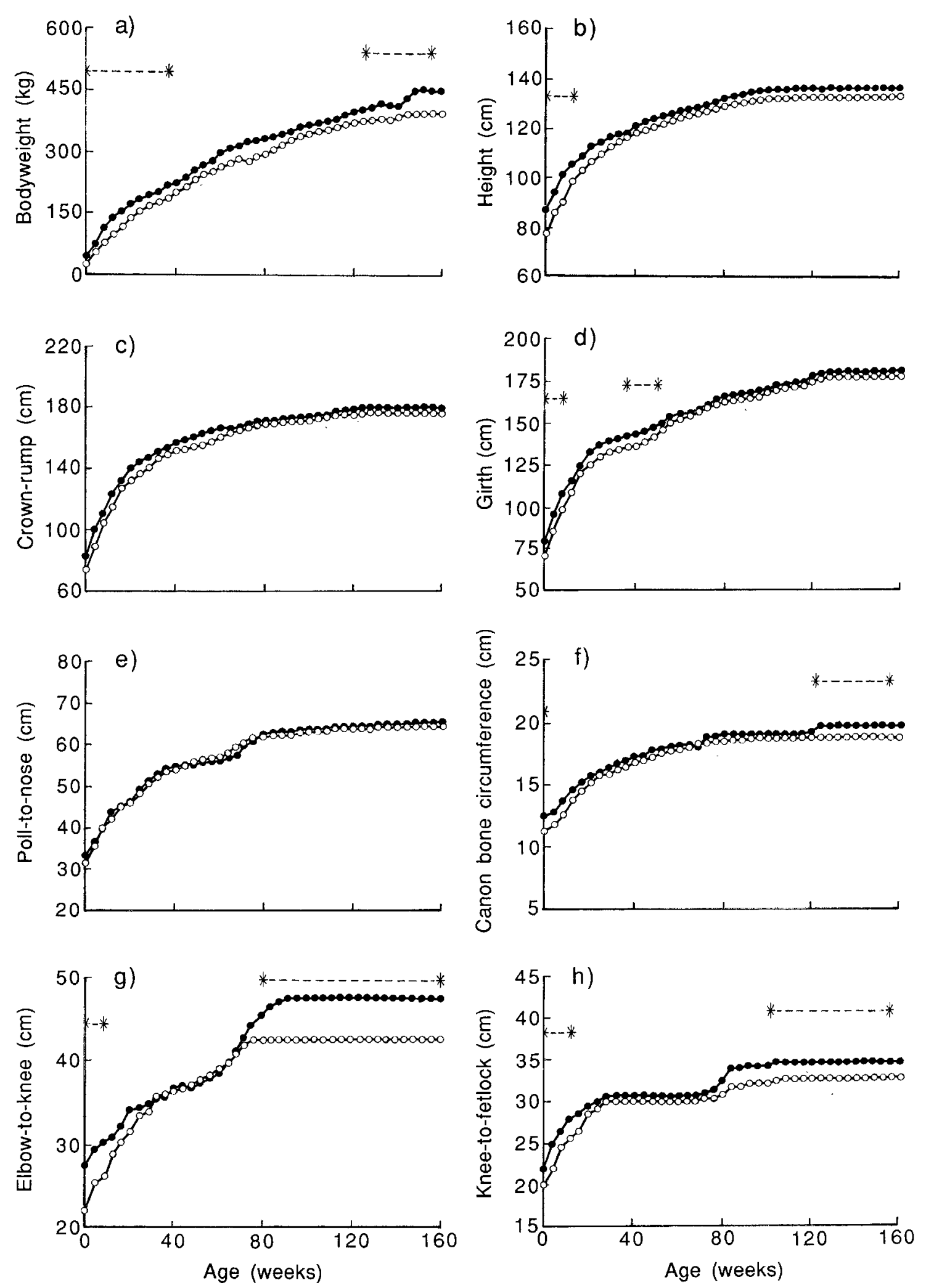

Figure 4 The mean growth curves exhibited by the P-in-Tb ('luxurious') animals and their P-in-P controls for (a) bodyweight, (b) height at the withers, (c) CR length, (d) girth, (e) poll-to-nose length, (f) cannon bone circumference, (g) elbow-to-knee length and (h) knee-to-fetlock length. The mean values between the asterisks differ significantly $(P<0.05)$ from each other. 

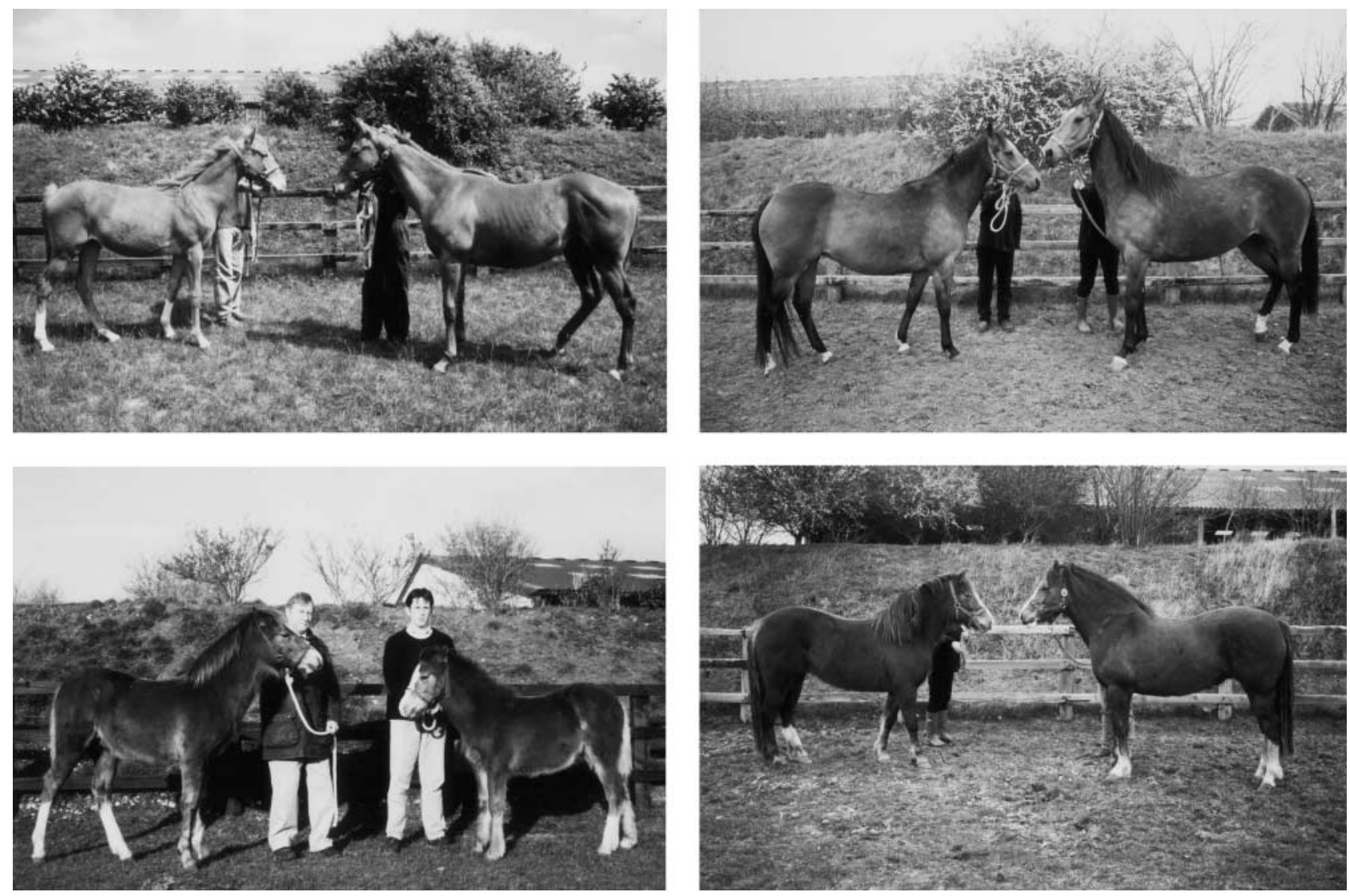

Figure 5 Photographs at 14 months of age of (a) a Tb-in-P 'restricted' yearling colt (left) together with a Tb-in-Tb control colt. The runting effect of the restricted uterine environment is apparent. (c) A P-in-Tb 'luxurious' yearling (left) compared with a P-in-P control. These two offspring were genetic full sisters and the considerable increase in body size occasioned by the 'luxurious' intrauterine environment is obvious. Photographs at 3 years of age comparing (b) a 'restricted' Tb-in-P filly (left) with a larger Tb-in-Tb control filly and (d) a larger 'luxurious' P-in-Tb filly (right) with a smaller P-in-P control filly.

for the 'luxurious' animals were higher than those of the P-in-P controls for bodyweight, height at the withers and girth, they tended to overlap for both the CR length and head length (poll-to-nose) measurements (Fig. $4 \mathrm{~d}$ and e). Bodyweight of the 'luxurious' offspring showed a pronounced increase compared with their controls during the last 20 weeks of the recording period (i.e. between 140 and 160 weeks of age).

The legbone profiles for the 'luxurious' animals showed some marked differences compared with those of their controls. For example, the cannon bone (knee-to-fetlock) increased sharply in length between 70 and 90 weeks of age, despite running remarkably parallel to the P-in-P profile, both before and after that sudden spurt in growth (Fig. 4h). In addition, the elbow-to-knee growth rate exhibited between 30 and 70 weeks of age in both groups continued for a further 10 weeks in the 'luxurious' animals (Fig. 4g). In addition, the circumference of the cannon bone showed two small but pronounced spurts compared with that of the P-in-P controls; one around 70 weeks in conjunction with the sudden increase in length of the bone and the second between 120 and 130 weeks in the absence of any change in length (Fig. 4f). Mean ages when longbone growth ceased were $78 \pm 10.1$ and $83 \pm 15.8$ for the cannon bone of the 'luxurious' and control foals respectively and $82.0 \pm 1.7$ and $72.7 \pm 4.4$ for the radius of the same two groups of foals.

The Ponderal index for the 'luxurious' foals showed a 3-4 week delay in the initial sharp post-partum rise compared with all three other groups of foals but then showed a pronounced rise, almost twice as high as the other groups, between 1 and 2 months of age; this perhaps reflected the very good muscle and fat deposition in these foals at birth as a consequence of their luxurious in utero environment. Thereafter, the curves remained essentially comparable to 140 weeks, although they crossed one another twice, at 40 and 60 weeks of age (Fig. 3b). It is apparent from both the Ponderal index (Fig. 3b) and Fig. 5 that the 'luxurious' progeny laid down considerable soft tissue, presumably fat, as they approached 3 years of age.

\section{Influences of donor and recipient}

Using two-way ANOVA to examine donor and recipient genotype influences (Table $1 \mathrm{~b}$ ) at birth, every measurement was influenced by recipient $(P=0.01)$; larger 
measurements were associated with a Tb recipient, even allowing for differences in donor genotype. The influence of the recipient was most striking at birth when the measurements were considered relative to the size they achieved at 3 years. However, donor genotype also influenced all the measurements $(P=0.01)$, except cannon bone circumference $(P=0.1)$. Once again, a Tb donor genotype was always larger than a $P$ genotype $(P<0.001)$. At 3 years, bodyweight, CR length, girth, cannon bone circumference and the lengths of both the radius and cannon bones were still influenced by both recipient and donor genotypes $(P<0.05)$, with higher figures being associated with a $\mathrm{Tb}$ genotype regardless of whether it was recipient or donor in origin. Height at the withers was now affected significantly only by donor genotype $(P<0.001)$. The percentage increase in measurements from birth to 3 years of age was affected only by the recipient with foals from $\mathrm{P}$ mares, irrespective of the donor genotype, showing larger increases than foals from Tb recipients $(P=0.01)$.

\section{Discussion}

Our results clearly support the original observations of Walton \& Hammond (1938) that a genetically large foal cannot reach its normal birthweight when gestated in a uterus that is smaller than normal, and the runting effect persists throughout life. Furthermore, our findings agree with those of Tischner (1985) and Tischner \& Klimczak (1989) that genetically small foals will be born heavier than usual if gestated in the uteri of larger than normal mares and, similarly, this increased size persists to adulthood.

The 'restricted' Tb-in-P foals in the present experiment suffered the double disadvantage of a smaller gross area of allantochorion combined with a reduced surface density of microcotyledons covering that smaller-than-normal exchange organ. In keeping with placentation in other species, most of the growth and development of the equine placenta occurs during the first half of pregnancy (Samuel et al. 1974, 1976, Steven 1982) whereas the majority of fetal growth takes place in the second half of gestation. Hence it is likely that, during the latter stages of pregnancy, the smaller-than-normal placenta would have found it difficult to keep up with the demands of the genetically driven $\mathrm{Tb}$ foal. In human pregnancies reduced nutrition in the second trimester could be expected to exert its maximum effect on body length, and food shortage in the third trimester would negatively affect the bodyweight of the fetus (Villar \& Belizan 1982). In a similar vein, Wallace et al. (2001), in their model of placentamediated restriction of fetal growth in over-nourished adolescent sheep, demonstrated that reductions in growth occurred only in late gestation. In the present restricted equine pregnancies the foals were born not only with reductions in bodyweight and body length (CR length) but in all measured parameters compared with their controls.
Conversely, the 'luxurious' P-in-Tb foals had the double advantage of a considerably larger gross area of allantochorion and a higher surface density of microcotyledons (Allen et al. 2002a). The combination led to a marked increase in the total microscopic area of fetomaternal contact at the placental interface compared with their P-in-P controls and these foals were born larger and very well covered.

The 'restricted' and the 'luxurious' environments produced foals with approximately $15 \%$ reduction or increase, respectively, in body dimensions. However, both the reductions and the increases declined to only $5 \%$ at 3 years of age. This decrease in the disparity between body dimensions as the offspring approached 3 years of age was occasioned by either an increased 'catch-up' growth rate in the Tb-in-P animals, or a curbed rate in the P-in-Tb animals. However, this discrepancy in growth rates between groups did not occur in a continuous manner throughout the 3 years of measurement and monitoring. Although they remained smaller or larger than their respective controls during the study, significant differences in most parameters had disappeared by 6 months of age. Increases in growth rates following periods of slowed growth from postnatal undernutrition have been demonstrated in ponies by Ellis \& Lawrence (1978) and it would appear from the present study that growth during the early postnatal period may likewise be governed by slowed or enhanced growth in utero.

Postnatal growth may be influenced by a host of conditions and circumstances. For the first few weeks of life the foal depends almost entirely upon its dam as the sole source of nutrients (Koterba 1990). Milk yield (kg/day) is normally much greater in Tb than P mares (Doreau \& Boulot 1989) and one would therefore have expected the Tb foals nursing from their $\mathrm{P}$ surrogate mothers to show a reduced weight gain compared with a Tb nursing from a Tb dam. Conversely, the P foals nursing on Tb dams could have been expected to increase their bodyweight at a greater rate than their P-in-P controls. Surprisingly, no significant differences existed between all four groups of foals when their mean weight increases during the first month of life were expressed as either $\mathrm{kg} /$ week or percentage increase in bodyweight. Their Ponderal indices indicated that all four groups received adequate milk since they all showed a sharp rise in the index associated with the bloom of suckling. Predictably, the highest index was seen in the $\mathrm{P}$ foals nursing on the Tb mares and the lowest was seen in the Tb foals nursing on the P mares. However, these differences had faded out by about 20 weeks of age, probably in reflection of the milk supply adjusting to the demands of the foal (Cymbaluk et al. 1993). Enhanced or slowed growth in the first 6 months appeared to be mainly through skeletal changes, evidenced by the changes in height, and this is not surprising in light of the early extension of the long bones in the horse.

Of the three major tissues, bone, muscle and fat, bone is the earliest to reach maturity so that height is determined 
early in life (Frape 1998). Since height at the withers is largely a reflection of linear growth of the long bones, the time of closure of the growth plates of these bones will determine the period up to when potential plasticity in height could be achieved. Growth plate closure in the foreleg has been reported to occur between 7 and 12 months in the cannon bone and between 24 and 30 months in the radius (Adams 1974). However, in the present study, cessation of long bone growth occurred later, at approximately 20 months of age, in both the radius and the cannon bone. Ellis \& Lawrence (1978) observed a marked delay in closure of the growth plates of the distal cannon bone and the first and second phalanges in weanling filly foals that had experienced a period of undernutrition. In our present experiment, by contrast, over- and undernutrition in utero, rather than postnatally, appeared to have no significant effect on the time of growth plate closure between the four groups. We noted two major spurts in longbone growth in all the animals in all four groups, which is contrary to the lack of any growth spurt reported previously for ponies by Campbell \& Lee (1981). Spurt-like growth phases of this nature were described by Heinze \& Lewis (1968) and it is likely that the growth spurts we observed, particularly the second one around 20-22 weeks of age, coincided with lush grazing in the Spring. Pagan et al. (1996) similarly reported growth rate increases in foals that were associated with season rather than age. The percentages of mature height and weight at different ages recorded in the P-in-P and Tb-in-Tb control offspring were similar to those reported previously by Hintz et al. (1979) and others.

Finally, the above discussion leads to the conclusion that neither the 'restricted' nor the 'luxurious' in utero growth experienced by these two experimental groups of foals had any major or long-lasting effect on the relative growth of the various body dimensions that were measured. Allowing for differences in uterine effects it was evident that the genotype of the foal influenced the postnatal size insofar as $\mathrm{Tb}$ animals were larger than $\mathrm{P}$ animals at both birth and 3 years of age. However, the changes that occurred in measured parameters during this time-period, judged as a percentage increase, were significantly influenced by the recipient, with foals from $\mathrm{P}$ uteri showing greater increases in all parameters with the exception of knee-fetlock length. Thus, the Tb carried by $\mathrm{P}$ mares were merely scaled down versions of the Tb carried by $\mathrm{Tb}$ mares. Conversely, the $\mathrm{P}$ carried by $\mathrm{Tb}$ mares were scaled up versions of the $\mathrm{P}$ carried by $\mathrm{P}$ mares. The measurements in the mature animals in each group, although not statistically significantly different, were, in all cases, of the order expected; namely, larger in the 'luxurious' animals than in their controls, and smaller in the 'restricted' animals than their controls, thereby indicating that the intrauterine effects on all the dimensions measured will persist throughout life.

\section{Acknowledgements}

This study was generously financed by The Horserace Betting Levy Board (Project No. 653), the Thoroughbred Breeders' Association and the Havemeyer Foundation of New York. The authors are grateful to Professor R V Short for enthusiastic stimulation and Dr Eurof Walters for help with statistical methods. Mr Edward Sear and Misses Caroline Turnbull and Sarah Watkinson kindly gave expert assistance with the rearing and handling of the foals.

\section{References}

Adams DR 1974 Lameness in Horses, edn 3. Philadelphia: Lea and Febiger.

Allen WR 1982 Embryo transfer in the horse. In Mammalian Egg Transfer, pp 135-154. Ed. CE Adams. Florida: CRC Press.

Allen WR, Wilsher S, Turnbull C, Stewart F, Ousey J, Rossdale PD \& Fowden A 2002a Influence of maternal size on placental, fetal and postnatal growth in the horse: I Development in utero. Reproduction 123 445-453.

Allen WR, Wilsher S, Stewart F, Ousey J \& Fowden A 2002b The influence of maternal size on placental, fetal and postnatal growth in the horse: II Endocrinology of pregnancy. Journal of Endocrinology 172 237-246.

Campbell JR \& Lee R 1981 Radiological estimation of differential growth rates of the long bones of foals. Equine Veterinary Journal $13247-250$.

Cymbaluk NF, Smart ME, Bristol FM \& Pouteaux VA 1993 Importance of milk replacer intake and composition in rearing orphan foals. Canadian Veterinary Journal 34 479-486.

Doreau M \& Boulot S 1989 Recent knowledge on mare milk production: a review. Livestock Production Science 22 213-235.

Ellis RNW \& Lawrence TJL 1978 Energy undernutrition in the weanling filly foal. I Effects of subsequent liveweight gains and onset of oestrus. British Veterinary Journal 134 205-211.

Frape DL 1998 Growth. In Equine Nutrition and Feeding, 2nd edn, pp 240-258. London: Blackwell Science Ltd.

Heinze CD \& Lewis RE 1968 Bone growth in the horse (Shetland pony) determined by orthopaedic markers. Proceedings of the 14th Annual Convention of the American Association of Equine Practitioners, pp 33-38.

Hintz HF, Hintz RL \& van Vleck LD 1979 Growth rate of Thoroughbreds. Effect of age of dam, year and month of birth and sex of foal. Journal of Animal Science $\mathbf{4 8}$ 480-487.

Koterba AM 1990 Nutritional support: enteral feeding. In Equine Clinical Neonatalogy, pp 728-746. Eds AM Koterba, WH Drummond and PC Kosch. Philadelphia: Lea and Febiger.

Pagan JD, Jackson SG \& Caddel S 1996 A summary of bone growth rates of Thoroughbreds in Kentucky. Pferdeheilkunde 12 285-289.

Rowell JG \& Walters DE 1976 Analysing data with repeated observations on each experimental unit. Journal of Agricultural Science $87423-432$.

Samuel CA, Allen WR \& Steven DH 1974 Studies on the equine placenta. I. Development of the micro-cotyledons. Journal of Reproduction and Fertility 41 441-445.

Samuel CA, Allen WR \& Steven DH 1976 Studies on the equine placenta. II. Ultrastructure of the placental barrier. Journal of Reproduction and Fertility 48 257-264.

Steven DH 1982 Placentation in the mare. Journal of Reproduction and Fertility 31 (Suppl) 41-55.

Thompson DW 1942 The rate of growth: the Ponderal index. In On Growth and Form, 2nd edn, pp 194-202. Cambridge: Cambridge University Press.

Tischner M 1985 Embryo recovery from Polish-pony mares and preliminary observations on foal size after transfer of embryos to large mares. Equine Veterinary Journal 3 (Suppl) 96-98. 
Tischner M 1987 Development of Polish-pony foals born after embryo transfer to large mares. Journal of Reproduction and Fertility 35 (Suppl) 705-709.

Tischner M \& Klimczak M 1989 The development of Polish ponies born after embryo transfer to large recipients. Equine Veterinary Journal 8 (Suppl) 62-63.

Villar J \& Belizan JM 1982 The timing factor in the pathophysiology of intrauterine growth retardation syndrome. Obstetrical and Gynecological Surveys 37 449-457.

Wallace J, Bourke D, Da Silva P \& Aitken R 2001 Nutrient partitioning during adolescent pregnancy. Reproduction 122 347-357.

Walters DE \& Rowell JG 1982 Comments on a paper by I Olkin and $M$ Vaeth on two-way analysis of variance with correlated errors. Biometrika 69 664-666.
Walton A \& Hammond J 1938 The maternal effects on growth and conformation in Shire horse-Shetland pony crosses. Proceedings of the Royal Society B 125 311-335.

Whitwell K 1997 Histopathology of grass sickness - comparative aspects of dysautonomia in various species (equine, feline, canine, leporids). Proceedings of the First International Workshop on Grass Sickness, Equine Motor Neurone Disease and Related Disorders, 1995, pp 18-20. Bern, Switzerland.

Received 1 July 2003

First decision 28 August 2003

Accepted 18 September 2003 